Reprod. Nutr. Dévelop., 1983, 23 (1), 115-127.

\title{
Ontogenèse gonadique et différenciation sexuelle du Loup Dicentrarchus labrax, en conditions d'élevage
}

C. ROBLIN, J. BRUSLÉ

Laboratoire de Biologie marine, Université de Perpignan, avenue de Villeneuve, 66025 Perpignan Cedex, France.

Summary. Gonadal ontogenesis and sex differentiation in the sea-bass, Dicentrarchus labrax, in fish culture conditions.

The histology of the different stages of gonadal development (appearance of PGC, edification of gonad primordium, organization of an undifferentiated gonad, testicular or ovarian development) has been studied in fingerlings and juveniles of sea-bass in fishculture conditions.

Sex differentiation with a caudo-cranial gradient was direct and more in accordance with length than with age. Ovarian and testicular differentiation occurred in fish 11 to 23 months old and from 90 to $187 \mathrm{~mm} \mathrm{SL}$. Testis ova were frequently observed.

\section{Introduction.}

Dicentrarchus labrax (Linnaeus, 1758), communément nommé Bar dans l'Atlantique et Loup en Méditerranée, est un poisson Téléostéen de l'ordre des Perciformes et de la famille des Serranidés. Cette espèce fréquente les zones côtières et les lagunes saumâtres et est commune sur le littoral du Golfe du Lion (Barnabé, 1976a ; Hervé, 1978 ; Roblin, 1980).

Des recherches ont porté sur sa reproduction en milieu naturel (BoulineauCoatanea, 1969 ; Barnabé, 1976a ; Caporiccio, 1976) ainsi que sur les techniques d'induction de ponte (Gandolfi et al., 1974 ; Alessio et al., 1976 ; Barnabé, 1976 ; Villani, 1976 ; Girin et Devauchelle, 1978).

Alors que les pratiques de reproduction artificielle sont devenues classiques dans le cadre des programmes d'élevage intensif et que l'aquaculture du Loup se réalise actuellement à l'échelle commerciale, il est curieux de constater que les modalités de son ontogenèse gonadique et de sa différenciation sexuelle $n$ 'aient jamais été étudiées.

Ce travail a été réalisé en vue de préciser, par voie histologique, la chronologie du développement de la gonade, en conditions d'élevage contrôlé. 


\section{Matériel et méthodes.}

Les alevins sont issus de pontes induites et selon la méthode décrite par Barnabé (1976b) et proviennent de deux écloseries : station de biologie marine et lagunaire de Sète et DEVA Sud du CNEXO à Palavas. Ils ont été ensuite placés en élevage dans des bassins dans les piscicultures de Font-Dame (MéditerranéePisciculture) et de Font-Extramer situées dans la partie S.O. de l'étang de SalsesLeucate et alimentées par des eaux de résurgence, tièdes $\left(17-18,5^{\circ} \mathrm{C}\right)$ et faiblement salées $(1,2$ à 1,6 p. 1000$)$.

L'âge des poissons est connu et exprimé en jours au cours des premiers mois. Les relations entre l'âge des alevins et des juvéniles et leur taille ainsi que* leur poids sont complexes. Elles dépendent non seulement des conditions d'élevage (Température : Guérin-Ancey, 1973 ; Pouliquen, 1974 - Salinité : Alliot et Pastoureaud, 1979 - Nourriture : Alliot et al., 1974) mais aussi de l'origine des géniteurs. Une très grande variabilité apparaît en effet à l'intérieur des divers lots en élevage ainsi que d'une année à l'autre. Les valeurs moyennes et maximales de croissance dimensionnelle et pondérale proposées par Girin (1979) pour les 3 premiers mois de vie sont données dans le tableau 1.

TABLEAU 1

\begin{tabular}{ccccc}
\hline \multirow{2}{*}{ Age (en j) } & \multicolumn{2}{c}{ Taille (Longueur totale) $(\mathrm{mm})$} & \multicolumn{2}{c}{ Poids $(\mathrm{mg})$} \\
\cline { 2 - 5 } & moyenne & maximale & moyenne & maximale \\
\hline 10 & 6 & 6 & 0,6 & 0,6 \\
20 & 9 & 9 & 6 & 6 \\
30 & 12 & 14 & 9 & 11 \\
40 & 16 & 18 & 25 & 80 \\
50 & 19 & 22 & 60 & 150 \\
60 & 21 & 23 & 95 & 400 \\
70 & 26 & 29 & 200 & 650 \\
80 & 34 & 37 & 500 & 900 \\
\hline
\end{tabular}

En ce qui concerne cette étude, les mesures de taille LS (longueur standard en $\mathrm{mm}$ ) et de poids ( $P$ en $\mathrm{mg}$ ou en $\mathrm{g}$ ), entre le $2^{\mathrm{e}}$ et le $20^{\mathrm{e}}$ mois d'élevage, sont très variables selon l'origine des lots (géniteurs atlantiques ou méditerranéens, écloseries de Sète ou de Palavas, années 1977 ou 1978). Cette grande hétérogénéité de croissance dimensionnelle et pondérale impose des tris répétés (avec élimination des "lots de queue »), les valeurs moyennes étant alors calculées à partir des échantillons sélectionnés dont les particularités physiologiques sont jugées les meilleures.

Les mesures moyennes établies au cours de ce travail (Roblin, 1980) sont données au tableau 2.

Les techniques histologiques (fixation: Bouin-Hollande, coloration: Azan) ont été appliquées à 127 exemplaires : alevins (LS $\leq 50 \mathrm{~mm}$ ) en coupes in toto, 
puis juvéniles (50 à $120 \mathrm{~mm} \mathrm{LS}$ ) et jeunes (de $120 \mathrm{~mm}$ à la taille de première maturité sexuelle) dont les gonades ont été prélevées.

TABLEAU 2

\begin{tabular}{|c|c|c|c|c|}
\hline \multicolumn{2}{|c|}{ Age en mois } & \multirow{2}{*}{$\begin{array}{c}\text { Taille (LS, } \mathrm{mm} \text { ) } \\
22,1 \pm 2,5\end{array}$} & \multicolumn{2}{|c|}{ Poids (P, mg ou g) } \\
\hline 2 & (1977) & & $164 \pm$ & $72 \mathrm{mg}$ \\
\hline & $\begin{array}{l}(1977) \\
(1978)\end{array}$ & $\begin{array}{l}31,3 \pm 4,6 \\
25,5 \pm 1,1\end{array}$ & $\begin{array}{l}552,5 \pm \\
283,8 \pm\end{array}$ & $\begin{array}{r}259,6 \\
32,3\end{array}$ \\
\hline & $\begin{array}{l}(1977) \\
(1978)\end{array}$ & $\begin{array}{l}38,0 \pm 4,8 \\
37,4 \pm 5,9\end{array}$ & $\begin{array}{c}1350,3 \pm \\
896 \pm\end{array}$ & $\begin{array}{l}764,2 \\
424\end{array}$ \\
\hline & $\begin{array}{l}(1977) \\
(1978)\end{array}$ & $\begin{array}{r}69,4 \pm 12,3 \\
56,2 \pm 1,9\end{array}$ & $\begin{array}{l}6372 \quad \pm \\
3096 \quad \pm\end{array}$ & $\begin{array}{r}3012 \\
487\end{array}$ \\
\hline 8 & (1977) & $84,9 \pm 16,2$ & $11624 \pm$ & 6560 \\
\hline & (1977) & $92 \pm 6$ & $15,3 \pm$ & $3,9(\mathrm{~g})$ \\
\hline & (1977) & $96 \pm 12,7$ & $16,3 \pm$ & 6,5 \\
\hline & (1977) & $111,2 \pm 12,6$ & $23,2 \pm$ & 5,8 \\
\hline & (1977) & $115,2 \pm 10,9$ & $25,1 \pm$ & 8,0 \\
\hline & (1977) & $113,4 \pm 13,1$ & $26,9 \pm$ & 7,9 \\
\hline & (1977) & $121,3 \pm 20,1$ & $27,5 \pm$ & 14,5 \\
\hline & (1977) & $126 \pm 5,6$ & $28,0 \pm$ & 1,4 \\
\hline 20 & (1977) & $146,5 \pm 12,0$ & $37,4 \pm$ & 14,9 \\
\hline
\end{tabular}

\section{Résultats.}

1) Apparition des CGP et mise en place des crêtes génitales. - Les coupes sériées pratiquées sur des alevins fixés aux $8^{\mathrm{e}}, 9^{\mathrm{e}}, 10^{\mathrm{e}}, 11^{\mathrm{e}}, 13^{\mathrm{e}}, 15^{\mathrm{e}}, 17^{\mathrm{e}}, 19^{\mathrm{e}}$ et $21^{\text {e }}$ jours $n^{\prime}$ ont pas permis d'identifier de cellules germinales.

Les toutes premières cellules germinales primordiales (CGP) sont décelables à partir du $23^{\mathrm{e}}$ jour, le long de la paroi cœlomique péritonéale, dans les zones correspondant aux territoires présomptifs des gonades : partie latérodorsale de la cavité viscérale postérieure, au voisinage des régions néphrétiques (rein : PI. I, fig. 1 ou canal de Wolff : PI. I, fig. 2), et de part et d'autre du mésentère dorsal. Ces cellules germinales, en petit nombre (1 à 2 par section), de forme circulaire ou ovalaire et d'un diamètre de 9 à $12 \mu \mathrm{m}$, sont disposées au contact de l'épithélium péritonéal (PI. II, fig. 1).

Une telle situation se rencontre du $23^{\mathrm{e}}$ au $36^{\mathrm{e}}$ jour. A partir du $37^{\mathrm{e}}$ jour, les CGP, toujours peu nombreuses, ne sont plus isolées et libres mais apparaissent entourées de cellules conjonctives (fibroblastes) ainsi que de fibres de collagène qui constituent un court pédicule (mésogonium) unissant le primordium gonadique à la paroi péritonéale (PI. II, fig. 2).

$\mathrm{Au} 43^{\mathrm{e}}$ jour, on note la mise en place d'une ébauche somatique continue ou crête génitale, faite de cellules somatiques et de fibres collagènes (PI. II, fig. 3), parmi lesquelles les CGP sont distribuées de façon discontinue.

Le $57^{\mathrm{e}}$ jour (PI. II, fig. 3), les cellules somatiques, plus nombreuses, entourent les cellules germinales dont le nombre s'est légèrement accru (de 2 à 4 par section); certains intervalles stériles des crêtes génitales se rencontrent encore. 

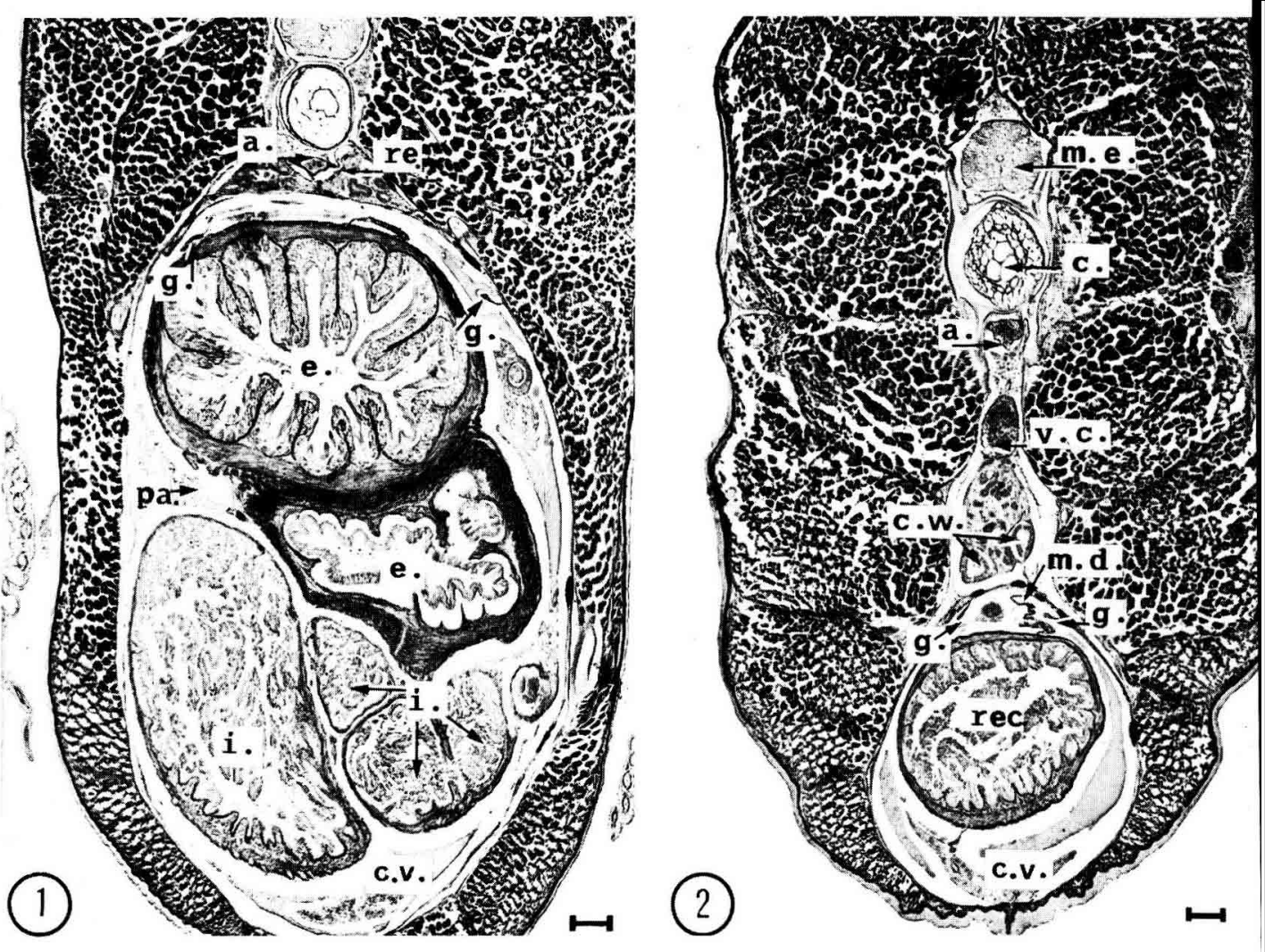

PLANCHE I

FIG. 1. - Coupe transversale d'alevin de Loup de 75 jours (25 mm LS) (niveau gastrique et intestinal) : les ébauches de gonade (g.) sont latéro-dorsales par rapport au tractus digestif (estomac : e et intestin : i).

FIG. 2. - Coupe transversale d'alevin de Loup de 75 jours $(25 \mathrm{~mm}$ LS) (niveau rectal) : les ébauches de gonade (g.) sont situées dorsalement par rapport au rectum (re.), de part et d'autre du mésentère dorsal (m.d.).

Echelle : $\longmapsto=100 \mu \mathrm{m}$.

Légende : a. : aorte ; c.g. : cellule germinale ; c.g.p. : cellule germinale primordiale ; c.p. : canal pneumatique ; c.py. : caecum pylorique ; c.s. : cellules somatiques ; c.v. : cavité viscérale ; c.W. : canal de Wolff ; e. : estomac ; $f$. : fibroblastes ; f.c. : fibres collagènes ; g. : gonade ; i. : intestin ; I.ov. : lamelłe ovarique ; l.t. : lumière de tubule séminifère ; m. : mélanocyte ; m.d. : mésentère dorsal ; m.e. : moelle épinière ; my. : myotome ; p. : pédicule gonadique ; pa. : pancréas ; p.p. : paroi péritonéale ; r. : rate ; re. : rein ; rec. : rectum ; spg. : spermatogonies ; st.c. : stroma conjonctif ; t.s. : tubule séminifère; v.c. : veine cardinale; v.n. : vessie natatoire; v.s. : vaisseaux sanguins. 
Entre le $68^{\mathrm{e}}$ et le $75^{\mathrm{e}}$ jour, le nombre des cellules germinales augmente progressivement mais leur distribution demeure discontinue au sein des ébauches dans lesquelles le stroma conjonctif s'enrichit en cellules somatiques tandis que le pédicule gonadique s'est allongé (PI. II, fig. 5).

2) Gonade indifférenciée. - Bien qu'aucune figure de division mitotique n'ait été décelée, le nombre des cellules germinales s'accroît régulièrement entre le $75^{\mathrm{e}}$ et le $140^{\mathrm{e}}$ jour. Les valeurs moyennes, calculées à chaque fois sur 5 poissons par comptage systématique sur toutes les coupes sériées, sont respectivement de 32 cellules à 78 jours, 53 à 86 jours, 71 à 91 jours, 105 à 99 jours, 178 à 116 jours et de 236 à 137 jours, mais il convient de noter de grandes variations individuelles. On constate, de plus, l'existence d'un gradient de distribution caudo-crânien, les cellules sexuelles étant plus nombreuses dans la zone postérieure des ébauches.

Sur le plan cytologique, ces CGP, d'un diamètre de 12 à $15 \mu \mathrm{m}$ sont de forme ovalaire ou sphérique, leur noyau est volumineux $(7 \mathrm{à} 9 \mu \mathrm{m})$ et un nucléole est bien individualisé. Elles sont dispersées, seules ou par petits groupes, au sein d'un stroma conjonctif (fibroblastes et fibres de collagène) bien développé (PI. II, fig. 6) et sont nettement réparties dans la zone corticale de la gonade dont les secteurs plus profonds sont occupés par des fibres collagènes denses et des vaisseaux sanguins, surtout développés au niveau de l'insertion gonadique (PI. III, fig. 1).

Une telle gonade indifférenciée se rencontre chez les alevins à partir du $4^{\mathrm{e}}$ mois $\left(>40 \mathrm{~mm}\right.$ LS) puis chez les juvéniles dont l'âge s'étale du $6^{\mathrm{e}}$ (LS $=56 \pm 2 \mathrm{~mm}$ ) au $8^{\mathrm{e}}$ mois (LS $=84 \pm 16 \mathrm{~mm}$ ) et parfois même jusqu'à 12-14 mois.

3) Différenciation ovarienne. - Sa discrimination repose sur deux types de critères, les uns cytologiques, les autres topographiques.

a) Cytologie : on observe d'abord la différenciation d'ovocytes (PI. III, fig. 2). Des ovocytes en début de méiose se rencontrent au sein de nids de cellules qui sont alors considérées comme étant des ovogonies; les différentes étapes de la prophase méiotique se déroulent de façon synchrone à l'intérieur de chaque groupe d'ovocytes.

De plus, des ovocytes en début de prévitellogenèse sont aisément repérables (PI. III, fig. 2) en raison de leur volumineux noyau (10-12 à 20-30 $\mu \mathrm{m}$ ) à nucléoles périphériques et de leur cytoplasme fortement basophile au sein des groupes d'ovogonies et d'ovocytes méiotiques. Cette phase d'accroissement ovocytaire n'est pas synchrone et divers stades de développement sont observables (le diamètre des ovocytes variant de 13-16 à 40-60 $\mu \mathrm{m}$ ).

b) Topographie : on note parallèlement à la différenciation cellulaire une organisation de lamelles ovariques au contact d'une cavité ovarienne (PI. III, fig. 3).

Une telle différenciation dans un sens femelle se rencontre entre le $12^{\mathrm{e}}$ et le $23^{e}$ mois et concerne des exemplaires de tailles variables (de 86-90 à $173 \mathrm{~mm}$ LS). Elle dépend plus de la taille que de l'âge du poisson mais la variabi- 

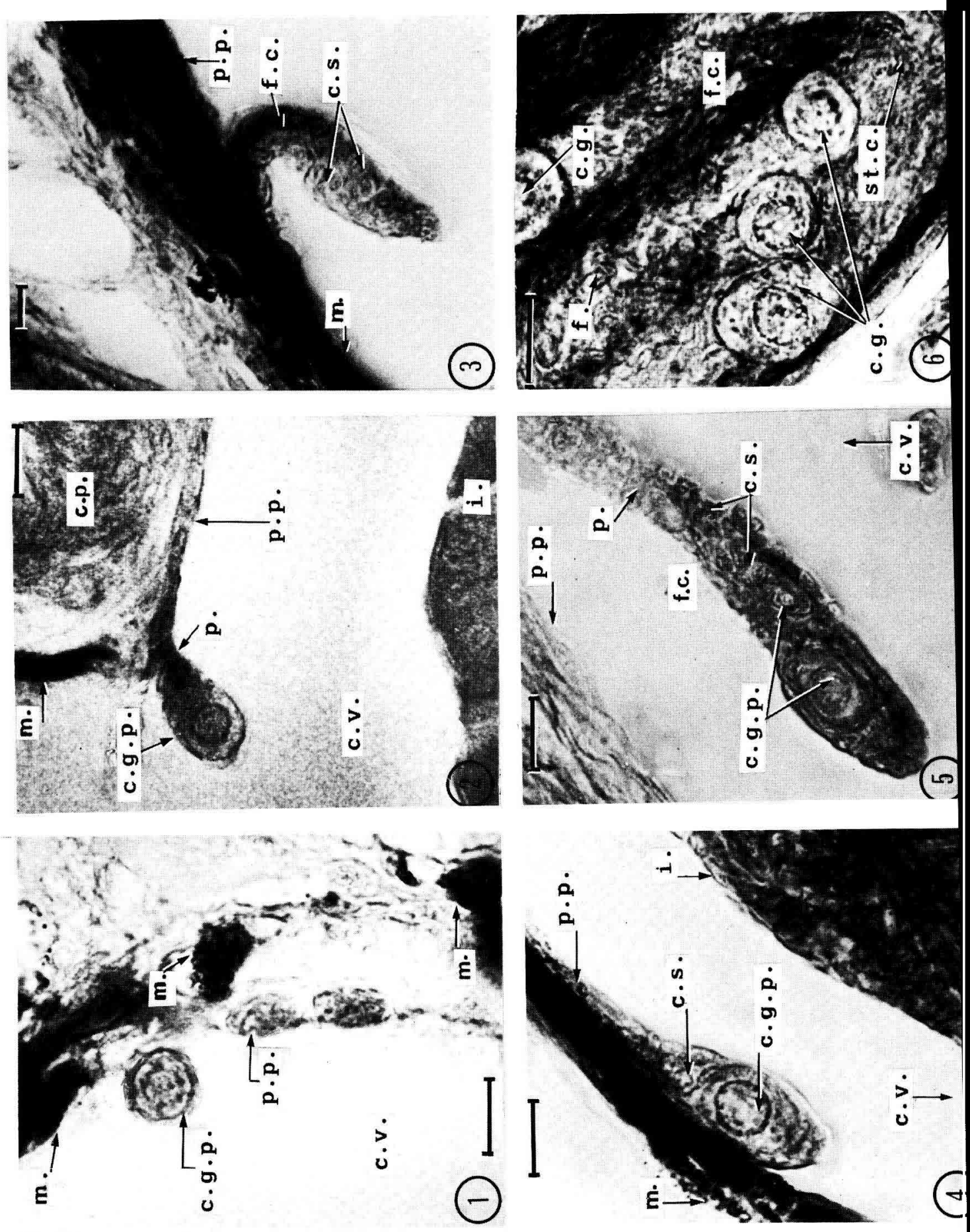
lité individuelle (de croissance et de gonadogenèse) demeure elle-même considérable.

4) Différenciation testiculaire. - Aucun critère cytologique ne peut être utilisé, la différence entre CGP, ovogonie et spermatogonie n'étant pas perceptible à l'échelle de la microscopie photonique. La différenciation dans le sens mâle repose donc uniquement sur des critères topographiques de distribution des cellules sexuelles et somatiques. II s'agit d'une organisation en nids (nids spermatogoniaux) séparés les uns des autres par des travées conjonctives riches en fibres collagènes et disposés surtout en situation corticale (PI. III, fig. 4). Les cellules considérées comme spermatogonies mesurent 11 à $16 \mu \mathrm{m}$, leur noyau est circulaire à un nucléole et présente un diamètre de 6 à $8 \mu \mathrm{m}$. Dans ces nids spermatogoniaux (PI. III, fig. 5), les figures mitotiques sont rares et on ne décèle aucune prophase méiotique ; ils évoluent ultérieurement en tubules constitués de cystes séminifères.

On remarque en outre la présence (PI. III, fig. 6), dans environ 50 p. 100 des cas de différenciation testiculaire et au sein des nids de spermatogonies, de petits ovocytes intratesticulaires d'un diamètre de 15 à $40 \mu \mathrm{m}$ et d'aspect cytologique normal. Ils sont le plus souvent nombreux, jusqu'à 10-15 par section de testicule.

II convient encore de noter que la différenciation du testicule débute dans la région postérieure de la gonade indifférenciée et qu'elle s'effectue selon un net gradient caudo-crânien. Les divers niveaux de section, de l'arrière vers l'avant, permettent alors de reconstituer les étapes de l'ontogenèse du testicule.

La différenciation dans le sens mâle est observable à partir du $12^{\mathrm{e}}$ mois dans les $2 / 3$ des exemplaires. Dans $1 / 3$ des cas, elle est soit plus précoce (dès 10 mois $1 / 2$, LS $=104$ à $133 \mathrm{~mm}$ ) soit au contraire beaucoup plus tardive (jusqu'au 33e mois et $187 \mathrm{~mm}$ LS).

\section{PLANCHE II}

FIG. 1. - $23^{e}$ jour $(10,6 \mathrm{~mm}$ LS) : une cellule germinale primordiale (c.g.p.) isolée et libre dans la cavité viscérale (c.v.) le long de la paroi péritonéale (p.p.).

FIG. 2. $-37^{e}$ jour $(14,8 \mathrm{~mm}$ LS) : cellule germinale primordiale (c.g.p.) reliée par un pédicule (p.) de fibres collagènes à la paroi péritonéale (p.p.) et formant le primordium gonadique.

FIG. 3. - 43e jour $(15,4 \mathrm{~mm}$ LS) : crête génitale stérile faite de cellules somatiques (c.s.) et de fibres collagènes (f.c.).

FIG. 4. - $57^{e}$ jour $(19,6 \mathrm{~mm}$ LS) : crête génitale au niveau d'une cellule germinale (c.g.p.) entourée de cellules somatiques (c.s.) et reliée à la paroi péritonéale (p.p.).

FIG. 5. - $68^{e}$ jour $(22,3 \mathrm{~mm}$ LS) : augmentation du nombre des cellules germinales (c.g.p.) et des cellules somatiques (c.s.) et allongement du pédicule gonadique ( $p$. ).

FIG. 6. - $91^{e}$ jour $(25,5 \mathrm{~mm}$ LS) : gonade indifférenciée : cellules germinales (c.g.) dispersées dans un stroma conjonctif (st.c.) contenant des fibroblastes (f.) et des fibres collagènes (f.c.).

Echelle : $\vdash-1=10 \mu \mathrm{m}$. 

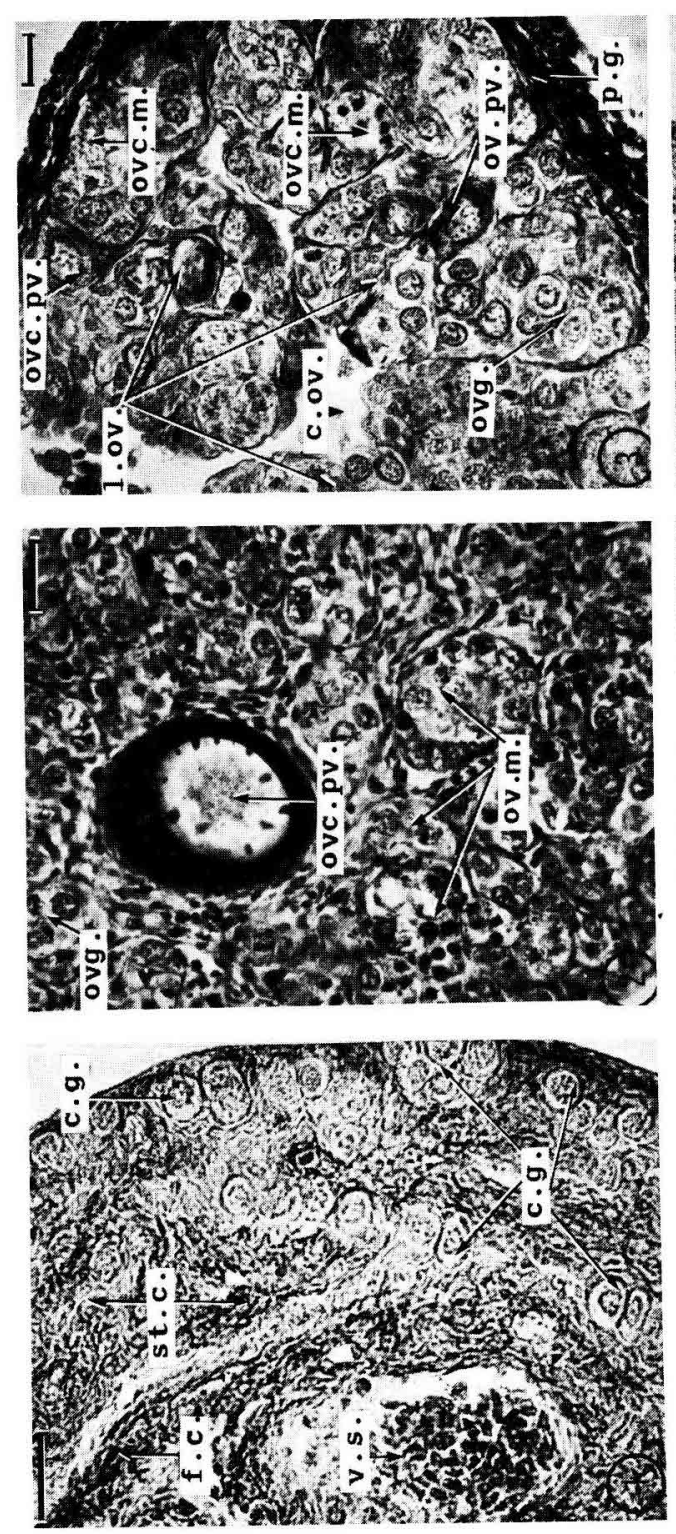
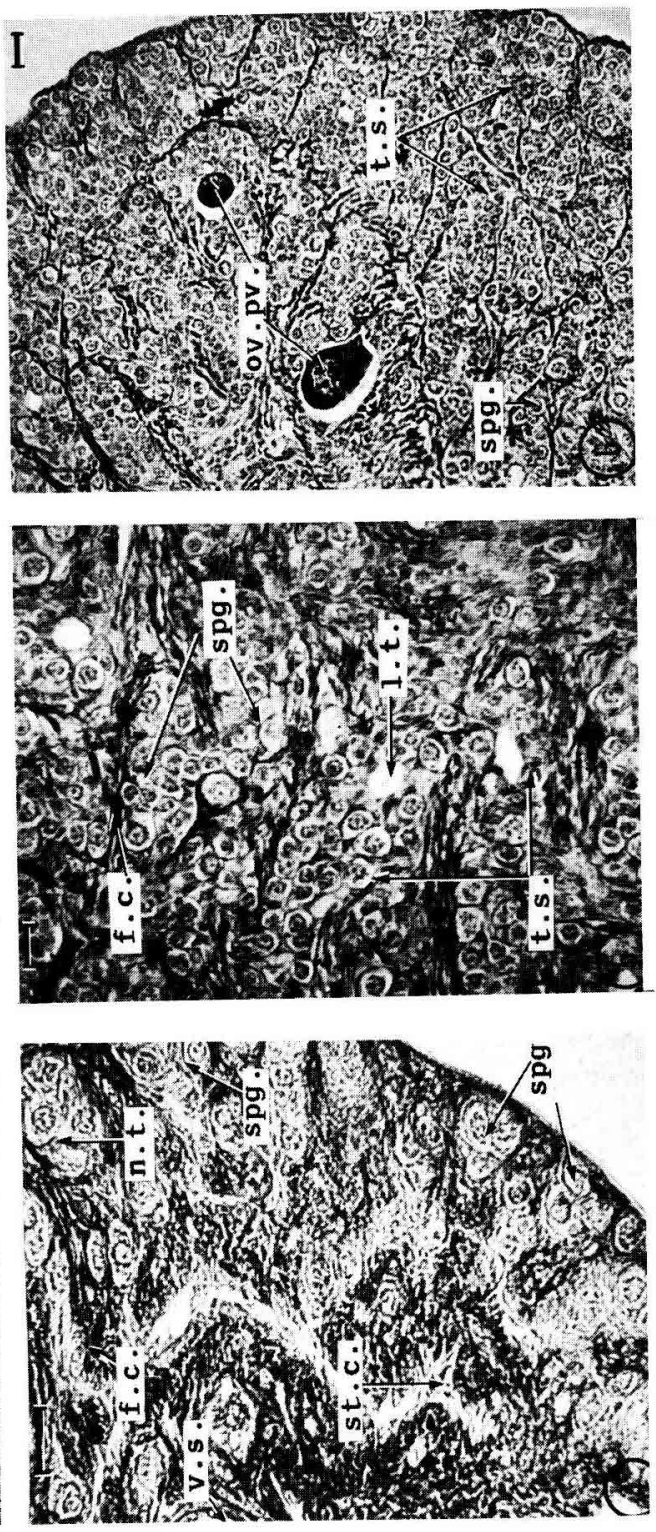
On note d'une façon générale une forte variabilité individuelle et tout retard de croissance est accompagné d'un retard de différenciation gonadique (puis d'un retard de gamétogenèse : Roblin, 1980).

\section{Discussion.}

La séquence des événements histologiques et cytologiques correspondant à l'édification d'un primordium gonadique, d'une crête génitale puis d'un ovaire ou d'un testicule a été reconstituée à l'aide d'un échantillonnage régulier et à peu près complet de poissons en conditions d'élevage définies et dont l'âge était connu avec précision.

Les premières étapes de mise en place de l'ébauche gonadique (primordium gonadique puis crête génitale) sont conformes aux descriptions jugées classiques réalisées dans diverses autres espèces (Perche : Mezhnin, 1978 ; Tilapia : Yoshikawa et Oguri, 1978 ; Truite : Lebrun, 1977 ; Takashima et al., 1980). L'ébauche somatique colonisée par les premières cellules germinales a pour origine l'épithélium cœlomique du mésoblaste somatopleural latéral, sans participation d'aucun élément du blastème néphrétique ni de l'interrénale donc sans organisation de type cortex-medulla (cf. revue de Lepori, 1980).

Les critères de sexualisation couramment utilisés sont les uns morphologiques, les autres cytologiques.

En l'absence d'une discrimination CGP-spermatogonie-ovogonie qu'il est impossible d'établir à l'échelle photonique, une diagnose précoce de ces diverses catégories cellulaires n'étant réalisable qu'au niveau ultrastructural (Satoh, 1974 ;

\section{PLANCHE III}

FIG. 1. - Gonade indifférenciée: cellules germinales (c.g.) par petits groupes dans le stroma conjonctif (st.c.) $\left(137^{\mathrm{e}}\right.$ jour, $\left.35 \mathrm{~mm} \mathrm{LS}\right)$.

FIG. 2. - Différenciation ovarienne : ovogonies (ovg.), ovocytes méiotiques (ov.m.) et ovocytes prévitellogénétiques (ov.pv.) (14e mois, $118 \mathrm{~mm}$ LS).

FIG. 3. - Différenciation ovarienne : les mêmes cellules (ovg., ov.m., ov.pv.) dans les lamelles ovariques (I.ov.) (12e mois, $113 \mathrm{~mm}$ LS).

FIG. 4. - Différenciation testiculaire: nids de spermatogonies (spg.) dans le stroma conjonctif (st.c.) riche en fibres collagènes (f.c.) $\left(12^{\mathrm{e}}\right.$ mois, $113 \mathrm{~mm} \mathrm{LS}$ ).

FIG. 5. - Différenciation testiculaire: spermatogonies (spg.) en nids séparés par des travées de fibres collagènes (f.c.) et début d'organisation en tubules séminifères (t.s.) avec une lumière des tubules (1.t.).

FIG. 6. - Ovocytes intratesticulaires: ovocytes prévitellogénétiques (ov.pv.) parmi les spermatogonies (spg.) des tubules séminifères (t.s.) (18 mois, $130 \mathrm{~mm}$ LS).

Echelle : $\longmapsto=25 \mu \mathrm{m}$. 
Bruslé et Bruslé, 1978 ; Bruslé, 1982), la reconnaissance d'une différenciation dans un sens testiculaire ou ovarien repose d'abord essentiellement sur des critères topographiques.

Ainsi, la différenciation ovarienne se traduit par la mise en place de lamelles ovariques orientées de façon centripète vers la cavité ovarienne. Simultanément se manifeste toutefois une différenciation ovocytaire qui concerne l'entrée en prophase méiotique puis l'accroissement prévitellogénétique. Cette entrée en méiose a été souvent considérée comme le meilleur critère de sexualisation dans un sens femelle (Nakamura et Takahashi, 1973 chez Tilapia; Satoh et Egami, 1973 chez Oryzias ; Takahashi, 1975, chez Poecilia ), par opposition aux testicules présomptifs dans lesquels aucune activité méiotique n'est décelable avant le début de la gamétogenèse.

La différenciation testiculaire se traduit uniquement par une augmentation progressive du nombre des cellules germinales qui s'organisent en nids disposés $\mathrm{d}^{\prime}$ abord en position corticale. Des travées de stroma et de fibres conjonctives séparent ces groupes de cellules contiguës à caractères cytologiques identiques et qui sont assimilées à des spermatogonies. II convient d'insister sur le caractère très progressif de cette édification testiculaire, à la fois dans le temps (phase de longue durée) et surtout dans l'espace (gradient postéro-antérieur). Ce gradient caudo-crânien impose d'ailleurs la nécessité d'effectuer des sections histologiques à divers niveaux, en particulier dans la zone postérieure. Un tel gradient a été également signalé chez la Perche par Mezhnin (1978) alors qu'au contraire chez Tilapia (Nakamura et Takahashi, 1973) la sexualisation s'effectue dans un sens céphalocaudal.

La mise en place des premières ébauches indifférenciées présente chez le Loup des analogies avec celle des autres Téléostéens tels que Tilapia (Nakamura et Takahashi, 1973) et Liza aurata (Bruslé, 1982). Cette gonadogenèse est lente et progressive et la phase d'indifférenciation se prolonge durant presque toute la première année de vie (classe 0 ).

En effet, les preuves histologiques et cytologiques de la sexualisation, c'està-dire de l'orientation dans un sens testiculaire ou ovarien, ne sont décelables qu'à la fin de la première année de vie (classe $0^{+}$) ou au début de la $2^{e}$ (classe 1).

On note d'ailleurs de très sensibles différences au sein de populations de même âge et de même origine. Cette forte variabilité individuelle se manifeste aussi bien en ce qui concerne la différenciation ovarienne (du $12^{\mathrm{e}}$ au $23^{\mathrm{e}}$ mois pour des tailles variant de $86-90 \mathrm{~mm}$ LS à $130-173 \mathrm{~mm}$ LS) que la différenciation testiculaire (du $11^{\mathrm{e}}$ au $23^{\mathrm{e}}$ mois et de 104 à $187 \mathrm{~mm} \mathrm{LS}$ ). Une telle variabilité se rencontre également chez la Carpe (Davies et Takashima, 1980).

Cette différenciation est directe, l'organogenèse ovarienne ou testiculaire faisant directement suite à un état indifférencié, situation qui est assez fréquente chez les Téléostéens (D'Ancona, 1956 ; Yamamoto, 1969) et qui s'oppose à celle des diverses espèces, telles l'Anguille (Kuhlmann, 1975; Colombo et Rossi, 1978, contredits d'ailleurs récemment par Bieniarz et al., 1981) et le Danio (Takahashi, 1977), chez lesquelles la différenciation est indirecte, faisant suite à une intersexualité transitoire ou hermaphrodisme juvénile. 
Cette différenciation est relativement tardive, du même ordre que celle décrite chez le Muge Liza aurata (Bruslé et Bruslé, 1978; Bruslé, 1982). Chez celui-ci toutefois, la différenciation ovarienne est plus précoce et précède dans le temps la différenciation testiculaire, ce phénomène ayant été également observé chez les Salmonidés (Ashby, 1957) et chez Tilapia (Nakamura et Takahashi, 1973) alors que chez le Loup aucune différence n'a été relevée, la sexualisation histologique étant à peu près synchrone entre mâles et femelles.

La différenciation gonadique dépend ici essentiellement de la taille du poisson et n'est pas directement fonction de son âge. Elle se produit pour le plus grand nombre à partir de $90-110 \mathrm{~mm}$ LS et tout retard de croissance se traduit par un retard de développement de la gonade. Il en est de même chez Tilapia (Dutta, 1979 cité par Shelton et al., 1981), chez l'Anguille (Kuhlmann, 1975 ; Bieniarz et al., 1981) où la différenciation sexuelle dépend de l'arrivée à une certaine longueur et chez la Truite (Lebrun, 1977) chez laquelle se manifeste une plus grande différence de développement des gonades entre poissons de même âge d'un même lot qu'entre ceux d'âges différents. Cette situation est contredite par Ashby (1957) chez la Truite et chez la Carpe par Davies et Takashima (1980) et confirmée par Takahashi et Takano (1971) chez Carassius où la gonadogenèse dépend de la durée en jours plus que de l'accroissement en taille. II convient pourtant d'insister (comme le fait d'ailleurs Vu-Tan-Tue, 1976) sur la valeur toute raltive de la notion d'âge, inhérente aux conditions d'élevage (caractères hydrologiques, température d'incubation et de développement, nourriture). Ainsi, dans une même station d'élevage (Font-Dame) et pour une même température $\left(16^{\circ} \mathrm{C}\right)$, des différences sensibles de croissance et de développement gonadique sont apparues (Roblin, 1980) en fonction de la salinité des eaux (les eaux de mélange à $12-13$ p. 1000 étant plus favorables que les eaux dessalées 1 à 2 p. 1000 de la résurgence).

On note enfin l'apparition précoce et généralement fréquente, dans les jeunes testicules, d'ovocytes ressemblant à ceux des jeunes ovaires. La présence d'ovocytes prévitellogénétiques dans une gonade indifférenciée ne saurait donc constituer la preuve cytologique d'une différenciation dans un sens femelle. De tels ovocytes intratesticulaires (ou testis ova), qui ont été par ailleurs observés chez d'autres espèces à différenciation directe telles que Tilapia (Yoshikawa et Oguri, 1978) et Brachydanio (Takahashi, 1977), traduisent une tendance à l'intersexualité juvénile. Cette potentialité est à rapprocher du fait que le Loup appartient à la famille des Serranidés chez lesquels les gonochoriques sont relativement rares (genres Dicentrarchus, Morone, Paralabrax, Roccus, Paranthias, Rypticus) et les hermaphrodites sont au contraire nombreux (hermaphrodisme synchrone de Serranus, Serranellus, Serraniculus, Bullisichthys, Diplectum, Hypoplectrus - hermaphrodisme successif chez Epinephelus, Centropristis, Mycteroperca, Chelidoperca, Cephalopholis et Pseudogramma - Atz, 1964 ; Smith, 1975). 
Remerciements. - Nous remercions très vivement Messieurs Paris et Cognie d'une part, Messieurs Conte et Fons d'autre part, qui ont permis la réalisation de ce travail.

\section{Références}

ALESSIO G., GANDOLFI G., SCHREIBER E., 1976. Induction de la ponte, élevage et alimentation des larves et des alevins des poissons euryhalins. Et. Rev. C.G.P.M., 55, 143157.

ALliot E., FeBVRe A., METAILleR R., PAStoureaud A., 1974. Besoins nutritifs du Bar Dicentrarchus labrax. Etude du taux de protéine et du taux de lipide dans le régime. Publ. CNEXO, Actes Coll., n ${ }^{\circ}$ 1, 215-231.

ALLIOT E., PASTOUREAUD A., 1979. Influence de la salinité sur la croissance et l'utilisation des aliments chez les loups juvéniles (Dicentrarchus labrax). Vie mar., 1, 13-17.

ASHBY K. R., 1957. The effect of steroid hormones in the brown Trout (Salmo trutta) during the period of gonadal differentiation. J. Embryol. exp. Morph., 5, 225-249.

ATZ J. W., 1964. Intersexuality in fishes. Am. Museum nat. History, New York, U.S.A.

BARNABÉ G., 1976a. Contribution à la connaissance de la biologie du Loup Dicentrarchus labrax (L.). (Poisson Serranidae). Thèse Doct. Etat. Sci. nat. USTL, Montpellier, 426 pp.

BARNABÉ G., 1976b. Rapport technique sur la ponte induite et l'élevage des larves du Loup Dicentrarchus labrax (L.) et de la Dorade Sparus auratus (L.). Et. Rev. C.G.P.M., 55, 63116.

BIENIARZ K., EPLER P., MALCZEWSKI B., PASSAKAS T., 1981. Development of European eel (Anguilla anguilla L.) gonads in artificial conditions. Aquaculture, 22, 53-66.

BOULINEAU-COATANEA F., 1969. Contribution à l'étude biologique du Bar, Dicentrarchus labrax (L.). Thèse $3^{e}$ cycle, Paris.

BRUSLÉ S., 1982. Contribution à la connaissance de la sexualité de Poissons Téléostéens marins gonochoriques (Mugilidés) et hermaphrodites (Serranidés). Th. Doct. Etat Sci. nat. Univ. de Perpignan, $360 \mathrm{pp}$.

BRUSLÉ S., BRUSLÉ J., 1978. An ultrastructural study of early germ cells in Mugil auratus Risso, 1810. Ann. Biol. anim. Biophys., 18, 1141-1153.

CAPORICCIO B., 1976. Etude ultrastructurale et cytochimique de l'ovogenèse du Loup IDicentrarchus labrax L.). Thèse $3^{e}$ cycle Biol. anim., Univ. Sci. Techn. du Languedoc, Montpellier, $101 \mathrm{pp} .+\mathrm{pl}$.

COLOMBO G., ROSSI R., 1978. Environmental influences on growth and sex ratio in different eels populations (Anguilla anguilla L.) of Adriatic coast. In Mc LUSKY D. S. et BERRY A. J. Physiology and behaviour of marine organism, 313-320.

D'ANCONA U., 1956. Morphogenèse et différenciation sexuelle chez les Poissons Téléostéens. Bull. Soc. Zool. France, 81, 219-229.

DAVIES P. R., TAKASHIMA F., 1980. Sex differentiation in common carp, Cyprinus carpio. J. Tokyo Univ. Fish., 66, 191-199.

GANDOLFI G., SCHREIBER B., ALESSIO G., 1974. Stato attuale della riproduzione artificiale di specie ittiche eurialine in Italia. Boll. zool., 41, 491-492.

GIRIN M., 1979. Méthodes de production des juvéniles chez trois poissons marins, le Bar (Dicentrarchus labrax), la Sole (Solea solea) et le Turbot (Scophtalmus maximus). Rapp. sci. techn. CNEXO, $\mathrm{n}^{\circ}$ 39, $202 \mathrm{pp}$

GIRIN M., DEVAUCHELLE N., 1978. Décalage de la période de reproduction par raccourcissement des cycles photopériodique et thermique chez des poissons marins. Ann. Biol. anim. Bioch. Biophys., 18, 1059-1066.

GUERIN-ANCEY O., 1973. Contribution à l'étude de la croissance des jeunes de Dicentrarchus labrax du golfe de Marseille. Cah. Biol. mar., 14, 65-77.

HERVE P., 1978. Ichthyofaunes comparées de deux étangs littoraux du Roussillon: SalsesLeucate et Canet-Saint-Nazaire. Ecologie générale et biologie de diverses espèces de poissons. Thèse Doct. $3^{e}$ cycle, Oceanogr. biol., Univ. Paris VI, 253 pp.

KUHLMANN H., 1975. Der Einfluss von Temperatur, Futter, Grösse und Herkurft auf die sexuelle Differenzierung von Glassaalen (Anguilla anguilla). Helgol. Wiss. Meeresunter., 27, 139-155. 
LEBRUN C., 1977. Différenciation sexuelle des gonades chez la Truite arc-en-ciel (Salmo gairdneri). Th. $3^{e}$ cycle, Univ. P. et M. Curie, $41 \mathrm{pp}$.

LEPORI N. G., 1980. Sex differentiation, hermaphroditism and intersexuality in vertebrates including man. Piccin medical books, Padua, 345 pp.

MEZHNIN E. I., 1978. Development of the sex cells in the early ontogeny of the common perch, Perca fluviatilis. I. Ichtyol., 18, 71-86.

NAKAMURA M., TAKAHASHI H., 1973. Gonadal sex differentiation in Tilapia mosambica, with special regard to the time of estrogen treatment effective in inducing complete feminization of genetic males. Bull. Fac. Fish. Hokkaido Univ., 24, 1-13.

POULIQUEN L., 1974. Contribution à l'étude de la croissance du juvénile de bars (Dicentrarchus labrax) élevés en milieu naturel. Publ. CNEXO, Actes Coll., n 1, 293-303.

ROBLIN C., 1980. Etude comparée de la biologie du développement lgonadogenèse, croissance, nutrition) du Loup Dicentrarchus labrax en milieu naturel et en élevage contrôlé. Th. Doct. $3^{e}$ cycle, USTL Montpellier, $272 \mathrm{pp}$.

SATOH N., 1974. Intercellular bridges between oocytes in the developing ovary of the teleost Oryzias latipes. Annat. zool. jap., 47, 215-220.

SATOH N., EGAMI N., 1973. Preliminary report on sex differentiation in germ celis of normal and transplanted gonads in the fish, Oryzias latipes. Genetics and mutagenesis of fish, SCHRODER J. H. Ed., Springer Verlag, 29-32.

SHELTON W. L., RODRIGUEZ-GUERRERO D., LOPEZ MACIAS J., 1981. Factors affecting androgen sex reversal of Tilapia aurea. Aquaculture, 25, 59-65.

SMITH C. L., 1975. The evolution of hermaphroditism in fishes, 295-310. In REINBOTH R., Intersexuality in the animal kingdom. Springer Verlag, Berlin, $446 \mathrm{pp}$.

TAKAHASHI H., 1975. Functional masculinization of female guppies, Poecilia reticulata, influenced by methyltestosterone before birth. Bull. jap. soc. scientif. fish., 41, 499-506.

TAKAHASHI H., 1977. Juvenile hermaphroditism in the Zebrafish, Brachydanio rerio. Bull. Fac. Fish. Hokkaido Univ., 28, 57-65.

TAKAHASHI H., TAKANO K., 1971. Sex hormone-induced precocious hypertrophy and ciliation of epithelial cells in the ovarian lumen of the Goldfish. Annat. zool. jap., 44, 32-42.

TAKASHIMA F., PATINO R., NOMURA M., 1980. Histological studies on the sex differentiation in rainbow trout. Bull. jap. scientif. Fish., 46, 1317-1322.

VILLANI P., 1976. Ponte induite et élevage des larves de Poissons marins dans les conditions de laboratoire. Et. Rev. C.G.P.M., 55, 117-132.

VU-TAN-TUE, 1976. Etude du développement du tube digestif des larves du Bar Dicentrarchus labrax. Arch. Zool. exp. Gén., 117, 439-509.

YAMAMOTO T. O., 1969. Sex differenciation. In HOAR W. S., RANDALL D. J., Fish Physiology, Acad. Press, NY, Vol. III, 117-175.

YOSHIKAWA $H$. et OGURI M., 1978. Sex differentiation in a cichlid, Tilapia zillii. Bull. jap. Soc. sci. Fish., 44, 313-318. 